\title{
Comunicación simultánea: un modelo para enfrentar los déficit lingüísticos del niño autista
}

\section{Juan Martos \\ Anunciación Mayordomo Socorro Puras}

Centro "Leo Kanner» de A. P. N. A.

\section{EL LENGUAJE EN EL NIÑO AUTISTA}

En la forma de desarrollar el lenguaje y en el uso del mismo, el niño autista es claramente diferente a los niños normales y a otros niños con trastornos del lenguaje. Recogemos aquí resumidamente las conclusiones más significativas sugeridas por algunos estudiosos del tema.

Kanner (1943) hacia referencia a las peculiaridades del lenguaje en el niño autista y las consideraba como esenciales para el diagnóstico del autismo. Existen graves problemas de comprensión del lenguaje, que se ven incluso reflejados en la escasa comprensión de la información transmitida por gestos, mímica, expresión facial, posturas del cuerpo, etc.; por otra parte, entre los niños que hablan, el lenguaje puede caracterizarse por una «ecolalia» (inmediata o retardada) con uso repetitivo, estereotipado y rígido de palabras y frases. Asimismo, existiría una confusión en el uso de formas directas, así como un escaso control del tono, volumen y entonación de la voz.

Para Rutter (1978), en casi todos los niños autistas se da un grave deterioro en la comprensión del lenguaje; no entienden aquellas instrucciones que están fuera de contexto o que encierran la combinación de dos o más ideas. Aproximadamente la mitad de los niños autistas, y especialmente aquellos que además tienen un retraso mental, no llegan a adquirir un lenguaje hablado provechoso, $y$ en aquellos que lo adquieren se dan 
algunas anormalidades, como la existencia de una ecolalia inmediata y repetición de frases estereotipadas durante el primer período del desarrollo del lenguaje. $\mathrm{El}$ lenguaje, en estos niños, no se usa en la forma usual para la comunicación social: no existe la conversación alterna entre emisor-receptor.

Otros autores, como Baltaxe y Simmons (1975), Wing (1975), Ricks y Wing (1975) y Baker y otros (1976), añaden a las anomalias del lenguaje anteriormente citadas la dificultad para la generalización del significado de las palabras y del lenguaje abstracto (uso de preposiciones, conjunciones, adverbios, etc.) y la disminución de comunicación gestual o simbólica.

En general, para la mayor parte de los investigadores actuales el niño autista tiene graves dificultades para seleccionar las reglas pragmáticas del lenguaje (uso del sistema); lo mismo acontecería respecto del uso inapropiado de los rasgos suprasegmentarios del lenguaje (entonación, ritmo, tono, intensidad, etc.) y del lenguaje no verbal (gestual) acompañante.

Este inquietante panorama ha dado lugar a diferentes alternativas de enfrentar la tarea de entrenar la creación del lenguaje (en aquellos sujetos en los que no existe) o mejorar las capacidades lingüísticas ya existentes. Lovaas (1981) ha informado de resultados satisfactorios utilizando procedimientos de condicionamiento operante. Sin embargo, el aspecto social y espontáneo del lenguaje parece siempre quedar muy por debajo de lo que pudiera considerarse una norma en la comunicación real del niño «normal». Siguen persistiendo esquemas rígidos de interacción lingüística $\mathrm{y}$, lo que es más importante, la competencia adquirida parece depender directamente de factores tales como el nivel de desarrollo global del sujeto y edad en que comienza el entrenamiento.

En cualquier caso, parece claro que existen tremendas dificultades para establecer lenguaje en niños autistas de nivel bajo.
Recientemente se está dando importancia a los procedimientos que utilizan técnicas de comunicación bimodal o simultánea (empleando al mismo tiempo palabra y gesto). En este sentido, hemos querido diseñar un programa de enseñanza del que se exponen algunos resultados preliminares. Permitasenos, sin que ello vaya a suponer un alargamiento excesivo, valorar las aportaciones de otros autores en este terreno.

\section{COMUNICACION SIMULTANEA}

"La comunicación simultánea y el "input" multisensorial mejoran el procesamiento de la información, la organización del pensamiento y experiencia y la conducta socioafectiva del niño autista». Esta hipótesis, planteada por Benaroya et. al. (1979) y desarrollada a través del entrenamiento de seis niños autistas con edades comprendidas entre los cinco y doce años, llegó a resultados que indican que los niños manifestaban una consistente adquisición del lenguaje de signos que en algunos casos fue transferido a las habilidades de comunicación verbal. Esta adquisición fue medida a través de la realización de sesiones estructuradas, que reflejaron un aumento en las vocalizaciones, y la combinación de signos de frases y, concretamente, el uso espontáneo de signos en cuatro niños, dos de los cuales eran funcionalmente mudos.

Un trabajo de Fulwiler y Fouts (1976) informa de que es posible la enseñanza del AMESLAN (lenguaje de signos americano) en un niño autista con graves perturbaciones de conducta. Estos autores apuntan la idea de que, cuando fracasan las técnicas convencionales de modificación de conducta (de tipo lovaasiano) de entrenamiento del lenguaje, el uso del lenguaje gestual, presentando simultáneamente palabra y signo, es apropiado para el desarrollo de la comunicación del niño autista. Una observación interesante que se desprende de este trabajo es la que hace referencia a que el lenguaje de signos ayuda a aprender a entender las 
expresiones faciales y a mantener el contacto ocular. Fulwiler y Fouts observaron cómo, después de veinte horas de entrenamiento, el niño autista aprendió los signos, elegidos previamente por el terapeuta, se produjo un incremento del habla vocal y se adquirió una sintaxis rudimentaria. También observaron cómo el uso espontáneo del AMESLAN se generalizó a otras situaciones y aumentó la interacción social.

En esta misma dirección hay que señalar el trabajo de Bonvillian, Nelson y Rhine ( $19^{8} \mathrm{I}$ ), que, en una espléndida revisión sobre la enseñanza del lenguaje de signos con niños autistas no hablantes, aportan datos de 100 niños indicando que prácticamente todos pueden aprender a usar los signos de forma comprensiva y expresiva y hacer combinación de ellos. Concluyen que las habilidades relacionadas con el habla pueden ser desarrolladas a través de un entrenamiento simultáneo utilizando palabras y signos.

A similares conclusiones llegan Konstantareas, Webster y Oxman (1979) en un estudio muy bien controlado, en el cual se entrena a cuatro niños autistas en un programa de comunicación simultánea en un periodo aproximado de ocho meses. El estudio que analiza el número y tipo de signos adquiridos evalúa los progresos de los niños en tres categorías lingüísticas: receptiva, reproductiva y elicitada; observándose cambios significativos en el aprendizaje del niño y efectos positivos sobre la interacción social evaluada en una situación de juego libre.

Aunque son más infrecuentes los trabajos que informan sobre la implicación de los padres en la utilización de programas de signos con sus hijos autistas, Casey (1978) ha elaborado un programa para padres -entrenados en el uso de los signos durante 20 sesiones de cuarenta y cinco minutos-, aplicado por ellos mismos en casa y completado con un entrenamiento en clase. Este programa consiguió un aumento de las conductas comunicativas (se usó una línea base múltiple como medida de contrastación). Por otro lado, el programa facilitó la generalización de la conducta comunicativa, manteniéndose las conductas adaptadas tres meses después de la iniciación del programa. Del estudio de Casey se desprende que la facilidad que los niños autistas presentan para la comprensión de gestos puede deberse al desarrollo más temprano en tareas de discriminación visual que en tareas de discriminación auditiva.

Finalmente existen un par de estudios que comparan la efectividad de tres tipos de tratamiento en el entrenamiento del lenguaje del niño autista. Brady y Smouse (1979) y Duverglas (1979). En ambos estudios se comparan tres métodos:

I. Verbalización o vocalización (entrenamiento tradicional tipo lovaasiano).

2. Lenguaje de signos.

3. Comunicación simultánea (que incluye el uso combinado de los dos tratamientos anteriores).

Los resultados de los dos estudios citados indican una sensible y efectiva superioridad del método de comunicación simultánea frente a los otros dos. Podemos concluir, siguiendo a Duverglas, que:

I. Existen diferentes interpretaciones teóricas acerca de las causas e implicaciones de los déficits del lenguaje en el niño autista.

2. Los problemas de lenguje son únicos en el niño autista y se extienden a todo el lenguaje hablado.

3. Existe una enorme variabilidad en las habilidades lingüisticas del niño autista.

4. El empleo de métodos operantes para entrenar el lenguaje espontáneo no ha resultado siempre en un uso funcional de lenguaje.

5. Parece que se obtienen mejores resultados, en el aspecto espontáneo, con el empleo de lenguaje de signos.

6. Los datos empíricos avalan la superioridad del método de comunicación simultánea.

7. La comunicación simultánea ayuda 
a entender las expresiones faciales y el mantenimiento del contacto ocular.

8. El entrenamiento en comunicación simultánea puede generalizarse a otras situaciones y favorecer la interacción social.

Animados por estas conclusiones, nos decidimos a poner en marcha un proceso de entrenamiento que detallamos a continuación:

\section{Método}

El estudio fue diseñado como experimento y tratamiento para comprobar la eficacia del uso del lenguaje gestual en niños autistas.

\section{Sujetos y marco}

El estudio fue realizado con dos niños diagnosticados como autistas, escolarizados en un centro dedicado al tratamiento de niños autistas y con problemas de la comunicación. Cada uno de ellos pertenece a distinta clase, siendo el criterio de asignación a la misma el nivel de desarrollo así como la edad de ambos. Cada clase está compuesta de un mínimo de tres y un máximo de cinco niños, estando al frente de la misma una terapeuta.

\section{Características de los sujetos}

Los sujetos eran un niño y una niña de 8,0 y de I I 2 años, respectivamente. Ambos poseen hermanos y el nivel económico de la familia es de tipo medio. La niña (sujeto I) presentaba un gran déficit a nivel cognitivo-verbal, que, medido en edad de desarrollo, correspondería a un año, siendo su nivel de desarrollo en otras áreas (motriz y social) de tres-cuatro años aproximadamente. La conducta de la niña se caracterizaba, en general, por cierto rechazo al contacto con el adulto, invarianzas ambientales, estereotipias con los dedos de la mano y utilización estereotipada de objetos.
El niño (sujeto 2) presentaba también un gran déficit a nivel cognitivo-verbal, que se concretaría en un nivel de desarrollo aproximado de un año, siendo ligeramente superior en el área motora y social (tres años). El comportamiento general del niño se caracterizaba por cierta inflexibilidad afectiva, alteraciones perceptivas, ciertas autroagresiones producidas por emociones (mordiscos en las manos), muecas corporales y estereotipias con los dedos de la mano.

En ambos niños existe ausencia de lenguaje expresivo, graves dificultades articulatorias, dificultades de comprensión y una gran dificultad para comprender y utilizar gestos. Con ambos niños se habían ensayado procedimientos tradicionales de enseñanza del lenguaje, en su aspecto receptivo, no obteniéndose ningún resultado concluyente.

\section{Procedimiento}

El objetivo del estudio en su primera fase consistió en entrenar a los niños en la comprensión de 24 órdenes sencillas verbales acompañadas de gestos, utilizando como metodología básica las técnicas de comunicación bimodal que recoge Marc Monfort en su libro Programa elemental de comunicación bimodal. Hemos seleccionado algunos ejemplos gráficos de estas órdenes:

Ambos niños poseían los requisitos necesarios para el entrenamiento: contacto ocular, atención en tareas, buen nivel de imitación y discriminación perceptiva.

Cada niño fue entrenado por su terapeuta en sesiones individuales de media hora de duración y durante un periodo de cuatro meses. Los pasos del programa fueron los siguientes:

I. Período de línea base inicial: el registro de la línea base consistió en computar durante tres días consecutivos el porcentaje de RRCC (respuestas correctas) que el niño ejecutaba una vez que el terapeuta emitía simultáneamente la orden verbal y su gesto correspondiente. Cada 

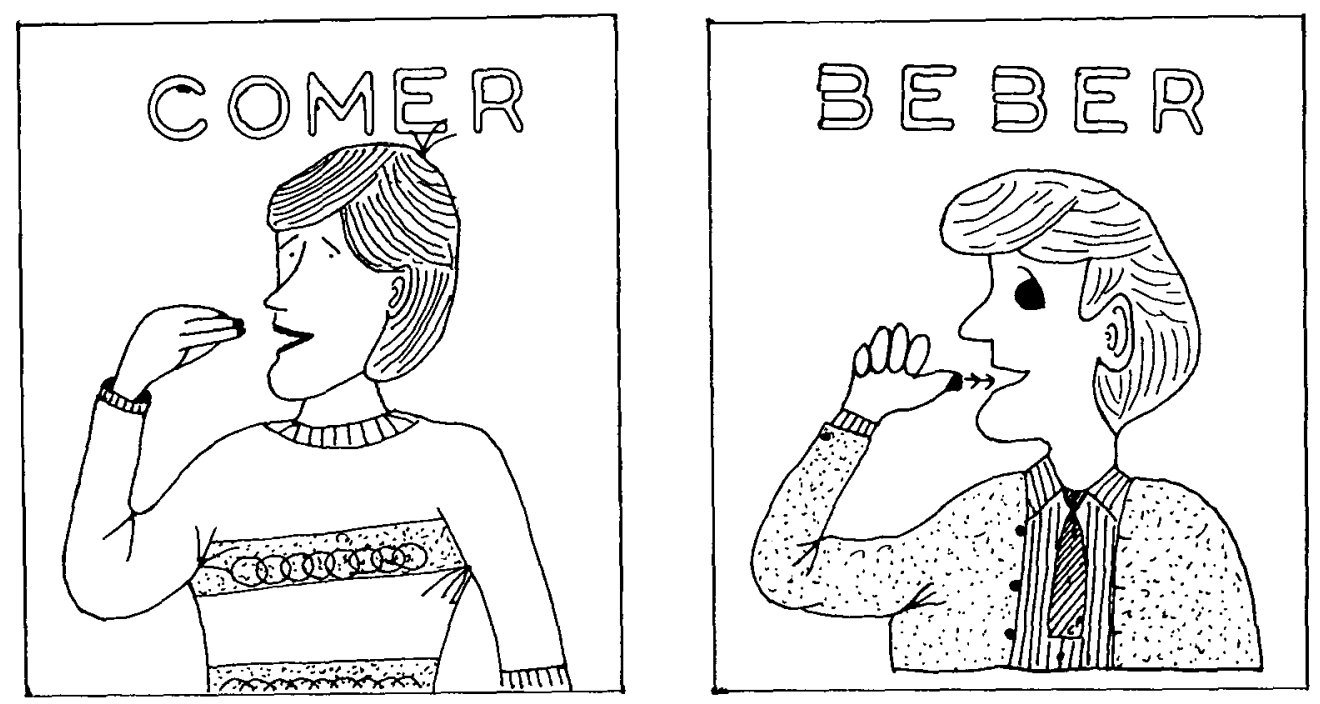

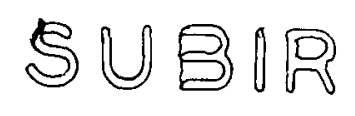

$B \Delta \circlearrowleft \Delta R$
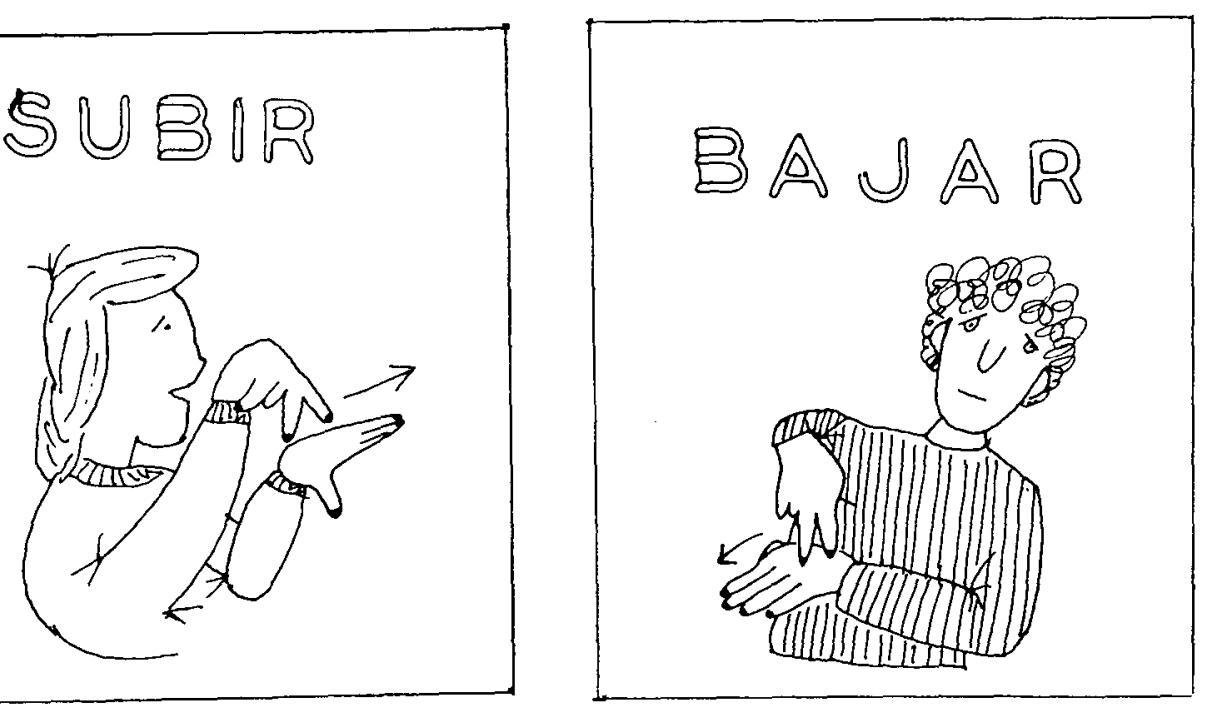

FIGURA I 
68

\section{Estudios}
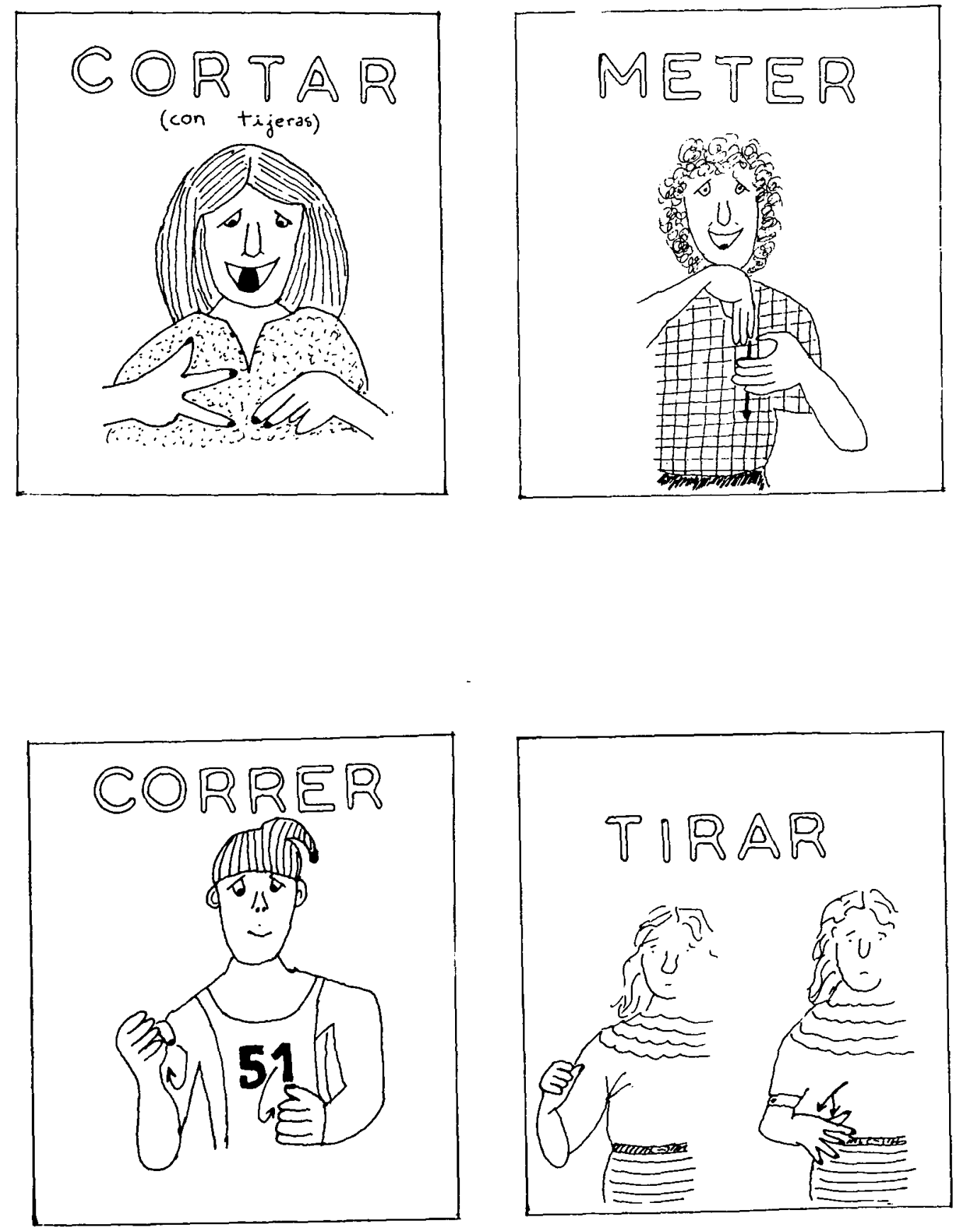

FIGURA 2 
orden se presentó dos veces y se tomó como criterio de respuesta correcta el cumplimiento de la orden en un periodo de tiempo no mayor de tres segundos. Durante esta fase no se empleó ningún tipo de reforzamiento.

2. Periodo de entrenamiento: el programa de entrenamiento propiamente dicho se inicia con la presentación de cinco órdenes, cuya probabilidad de respuesta correcta era mayor que otras, puesto que el niño comprendía algunas de ellas (se utilizó como criterio de selección de las cinco órdenes el registro de la línea base inicial); si el niño no efectuaba alguna de las mismas, se empleó un instigador físico, que se iba reduciendo gradualmente hasta desaparecer. A continuación se introducen nuevas órdenes con las que se seguia el mismo procedimiento. Una vez adquiridas estas últimas, se rotaban con las anteriores, es decir, se le presentaban al niño, en una misma sesión, todas las órdenes trabajadas hasta el momento. Así se continuó hasta la finalización del programa. En esta fase se administró recompensa social y primaria después de cada una de las órdenes ejecutadas.

3. Periodo de evaluación: como medidas de evaluación, al final del entrenamiento, se realizaron:

a) Una línea base final con gesto para comparar la eficacia o no del programa de entrenamiento.

b) Una linea base final sin gesto para observar el grado de dependencia que cada sujeto tenía del gesto.

c) Para comprobar el nivel de generalización, se introdujeron para ambos sujetos cinco órdenes nuevas, aumentándose datos a lo largo de tres sesiones.

En esta fase no se administró ningún tipo de reforzamiento.

\section{Registro}

Todas las sesiones eran registradas en hojas estandarizadas en las que iban especificados el nombre del niño, la fecha de la sesión, quién la registró, las órdenes empleadas como estímulo discriminativo (E-d), así como las respuestas correctas (RC), las respuestas incorrectas (RI) y las ayudas (Ay) o instigación física.

Como respuesta correcta, consideramos la ejecución de la orden emitida por el terapeuta en un periodo no mayor a tres segundos. La respuesta incorrecta fue considerada como aquella en que el periodo de latencia era mayor a tres segundos o bien cuando el sujeto confundía la orden dada. La ayuda o instigación física consistió en que el terapeuta realizaba, conjuntamente con el niño, la orden emitida o cuando aquél señalaba el objeto del cual se tenía que valer el niño para ejecutar la orden.

\section{Resultados}

Los resultados del sujeto 1 vienen $\mathrm{da-}$ dos en 43 sesiones:

- 3 de línea base.

- $3 \mathrm{r}$ de entrenamiento.

- 3 de línea base final con gesto.

- 3 de linea base final sin gesto.

- 3 de generalización.

Los resultados del sujeto 2 vienen $\mathrm{da}$ dos en 59 sesiones:

- 3 de línea base.

- 45 de entrenamiento.

- 4 de linea base final con gesto.

- 4 de línea base final sin gesto.

- 3 de generalización.

Partimos, en ambos sujetos, de una linea base; en el sujeto I con un porcentaje del 21,3 por 100 de RRCC, y en el sujeto 2 , con un porcentaje del i 8 por 100 de RRCC a las 24 órdenes. Nuestro objetivo se consideraba logrado si los sujetos llegaban a alcanzar el porcentaje de RRCC del 85 por 100 . Cada paso del programa consistía en el entrenamiento de órdenes nuevas o de rotación de órdenes adquiridas, siendo también el criterio para la introducción de un nuevo paso la realización de las órdenes en un 85 por 100 de los ensayos presentados, con una 


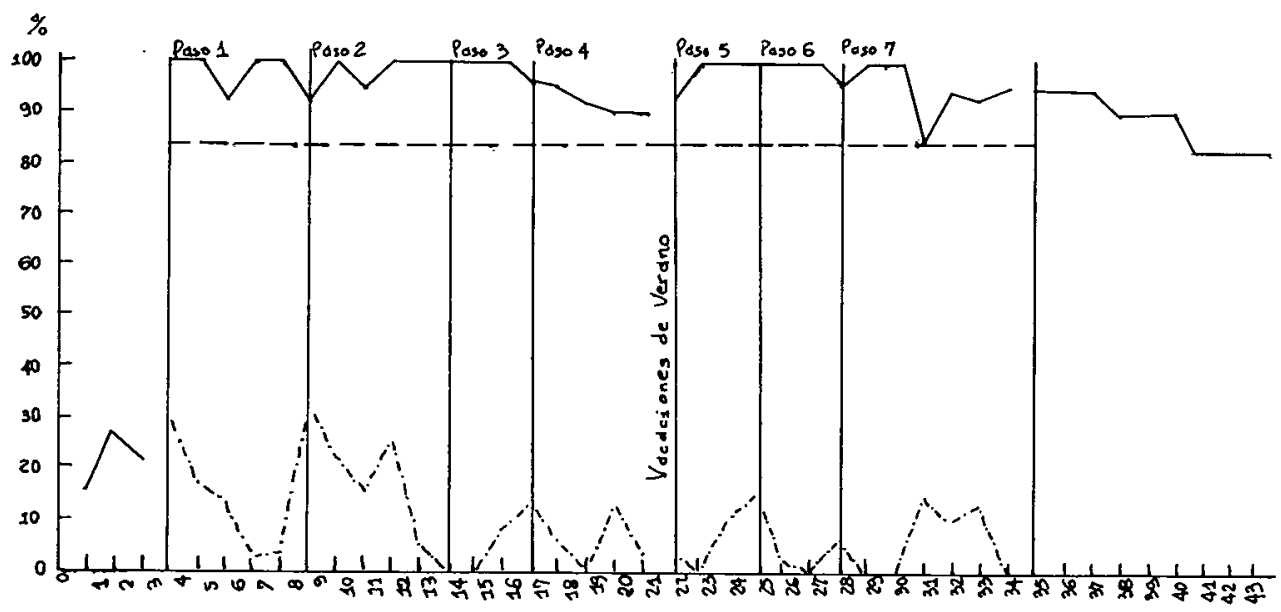

FIGURA 3

ayuda máxima del ıo por ıoo. El número de sesiones para cada paso variaba según la dificultad que el niño encontraba para llevar a cabo la orden presentada.

En el programa de entrenamiento se observan dos periodos:

I. Anterior a las vacaciones de verano, constando de is sesiones en el sujeto 1 y de 22 sesiones en el sujeto 2. En este periodo se llevaron a cabo los cuatro primeros pasos del programa.

2. Posterior a las vacaciones, constando de 13 sesiones en el sujeto I y de 22 sesiones en el sujeto 2 .

En ambos sujetos hay que destacar ciertas diferencias. En el caso del sujeto I, en el período anterior a vacaciones el porcentaje de ayuda es superior. Esto puede ser debido a que el comienzo del entrenamiento tuvo cierta dificultad de comprensión de la tarea. En el caso del sujeto 2, sin embargo, se percibe que el porcentaje de ayuda era inferior en el primer período de entrenamiento (antes de las vacaciones), y esto se puede explicar por el hecho de que las órdenes trabajadas en ese período eran más fami- liares para el sujeto debido a la naturaleza de la tarea. Además, es de destacar que el porcentaje de RRCC es más alto que en el periodo posterior.

En el segundo periodo, posterior a vacaciones, en el sujeto 1 el nivel de ayuda se mantuvo más estable, y se consiguieron más órdenes en menos sesiones, debido a que la niña llega a comprender la tarea. En el sujeto 2, las ayudas aumentan justo al volver a retomar el entrenamiento y, además, la media de RRCC es ligeramente inferior al periodo anterior de vacaciones. Es de resaltar, a pesar de este resultado, que en este período el sujeto conseguía las órdenes más rápidamente, aunque eran introducidas más dentro de cada paso y no eran conocidas.

Al final del entrenamiento se hicieron dos líneas base, una con gesto y otra sin gesto. En el caso del sujeto I, la línea base con gesto se realizó durante tres dias, y el porcentaje de RRCC fue del 95 por ioo. En el caso del sujeto 2, la línea base se realizó durante cuatro dias, y el porcentaje de RRCC fue del 96 por 100. la línea base del sujeto I sin gesto a lo largo de tres días arrojó un 90 por $100 \mathrm{de}$ 


\section{Estudios}

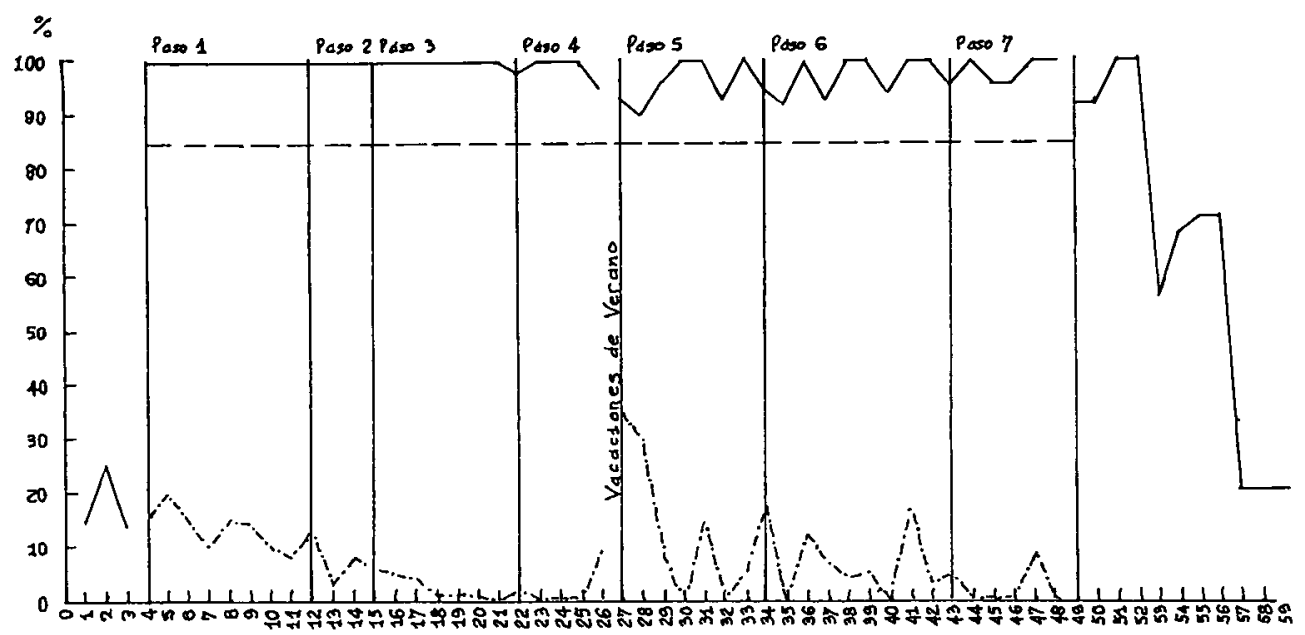

FIGURA 4

RRCC, y en el caso del sujeto 2 a lo largo de cuatro dias arrojó un porcentaje de RRCC del 65,75 por 100.

Con respecto a la generalización, el porcentaje de RRCC en el caso del sujeto I fue de un 80 por 100 , y en el caso del sujeto 2 el porcentaje de RRCC fue de un 20 por 100.

\section{Discusión}

Los resultados obtenidos confirman nuestros ánimos y están de acuerdo con las conclusiones a las que hacía referencia Duverglas. En ambos sujetos se ha operado una comprensión significativamente mayor de las órdenes entrenadas si comparamos el período de línea base inicial y el periodo de evaluación final. La utilización simultánea de palabra gesto ha redundado en beneficio de ambos sujetos, fomentando de paso un mayor rendimiento en tareas de atención e imitación. Una observación interesante y común a ambos sujetos es cómo, a medida que transcurría el entrenamiento, se constataba un mejor aprendizaje de las órdenes, aunque en cada paso del programa se incluyera mayor cantidad de ellas. La instigación física o señales que se utilizaban como ayuda decrecen rápidamente en el transcurso del programa. La constatación de que, aun cuando se retira el gesto, se mantiene un nivel óptimo (del 90 por 100 en el sujeto I y del 65,75 por 100 en el sujeto 2) de ejecución, valida los procedimientos de utilización de comunicación simultánea, al menos como soporte básico, fundamental y complementario para establecer un mayor nivel de comprensión en el niño autista. Las diferencias observadas en cuanto a dependencia del gesto en ambos sujetos, parecen estar en relación, por un lado, con el nivel de discriminación perceptivo y, por otro, con la comprensión de la tarea a realizar. Desde este punto de vista, y como veíamos antes, el gesto puede ayudar significativamente a una mejor ejecución de las tareas propuestas.

Los datos que se aportan en el periodo de generalización son importantes y a tener en cuenta. Al menos en uno de los sujetos se ha operado significativamente una buena comprensión de órdenes no entrenadas previamente.

Quizá el aspecto más interesante, que no refleja este articulo, pero que ha empezado a ocurrir con cierta frecuencia en uno de los sujetos, es la utilización de gestos en situaciones funcionales como medio de comunicación con el terapeuta. 
Aquí juega, evidentemente, un papel muy importante la imitación y, en concreto, la posibilidad de realizar imitación diferida que presupone ya la existencia de imágenes mentales concretas con contenido simbólico. Este puede ser el primer paso para que el niño autista establezca comunicación con el entorno que le rodea $y$ salga del aislamiento al que su enfermedad le tiene sometido.

\section{Referencias}

Baker, L.; Cantwel., D.; RUtTer, M., y Bartak, E.: «Language and Autism». En Ritvo, E. (Eds.): Autism: Diagnosis current research and management. Nueva York. Spectrum, 1976.

BAltAXE, A. M., y Simmons, J. A.: "Language in childhood psychosis: a review». Journal of speech and hearing disorders, 1975, 40, 439.

Benaroya, S.; Wesiey, H.; OGll.vie, L. S.; KLein, y Clarke, E.: «Sing language and Multisensory input training of children with communication and related developmental disorders». Journal of astism and developmental disorders, vol. 9, núm. 2, 1979.

BONVILliam, J. D.; Nelson, R. E., y RhYNE, J. M.: "Sing language and autism». Journal of autism and developmental disorders, vol. i I, núm. I, $198 \mathrm{I}$.

BRADY, D. O., y SMOUSE, A. D.: «A simultaneous comparison of three Methods for language training with an autistic child: an experimental single case analysism. Journal of autism and childhood schizopbrenia, vol. 8, núm. 3, 1978.

CASEY, L. O.: "Development of communicative behavior in autistic children: a pavent program using manual signsm. Journal of autism and childhood schizophrenia, vol. 8, núm. 1, $197^{8}$.

DUVERGI.AS, G.: «Comparison of three language trainig methods in mute autistic children». University of Washington. Seatle, 1979 .

FULwILER, R. L., y FOUTS, R. S.: «Acquisition of American sign language by a Noncommunicating autistic child». Journal of autism and childhood schizophrenia, vol. 6, núm. 1, 1976.

KANNER, L.: "Autistic disturbances of affective contact». Nervous child. 1943, 2, 21 7-290.

Konstantareas, M. M.; Nebster, C. D., y OXMAN, J.: «Át alternative to speech training: simultaneous communication". Journal of child psycbology and psychiatry, 1979.

LovaAs, O. I.: The autistic child. Irvington Publishers. Inc. 1979. Nueva York. Traducción castellana: El niño autista. Edit. Debate, $198 \mathrm{I}$. Madrid.

MONFORT, M.; ROJO, A., y JUÁRez, A.: Programa elemental de comunicación bimodal. Ed. CEPE, 1982.

Polaino, A.: Introducción al estudio científico del autismo infantil. Ed. Alhambra Universidad. Madrid, 1982.

RICKS, D. M.: «Vocal communication in pre-verbal normal and autistic children». En O'CONNOR, N. (ed.): Language, cognitive deficits and retardation. Butterworths. Londres, 1975.

RIVIĖRE, A., y BELLINCHÓN, M.: «El lenguaje en el niño autistam. Infancia y aprendizaje., vol. 13, julio 1981.

RUTTER, M.: "Language disorder and infantile autism». En Autism, M. RUTTER y E. SCHOPLER (Ed.): Plenun Press, $85-105,1978$.

WING, L.: "Study of language impairments in serely retarded children». En O'CONNOR, N. (Ed.): Language, cognitive deficits and retardation. Butterworths. Londres, 1975.

WING, L.; EVERARD, M. P., y otros: Autismo infantit. Aspectos médicos y educativos. Ed. Aula XXI. Santillana. Madrid, 1982.

Webster, C. D.; McPherson, H.; Sioman, L.; Evans, M. A.; Kuchar, E., y Fruchter, D.: "Gestures as a means of communication with an autistic boy: a case study". Journal of autism and childhood schizopbrenia, 3, 337-46, 1973 .

\section{Resumen}

El empleo de procedimientos de comunicación simultánea, en el tratamiento de los niños autistas, es una de las vias más prometedoras para incrementar la competencia comunicativa de estos niños. En este articulo se revisan los resultados del tratamiento de dos niños autistas con métodos de comunicación simultánea y las implicaciones de este método para el tratamiento de los niños autistas.

\section{Summary}

The use of simultaneous communication methods for the treatment of autistic children is one of the most promising ways for increasing the communicative competence of these children. In this paper, the results of the treatment of two autistic children with simultaneous communication methods and its implications are revised. 